\title{
FINITE AMPLITUDE THERMAL CONVECTION WITH VARIABLE GRAVITY
}

\author{
D. N. RIAHI and ALBERT T. HSUI
}

(Received 17 March 2000)

\begin{abstract}
Finite amplitude thermal convection is studied in a horizontal layer of infinite Prandtl number fluid with a variable gravity. For the present study, gravity is restricted to vary quadratically with respect to the vertical variable. A perturbation technique based on a small parameter, which is a measure of the ratio of the vertical to horizontal dimensions of the convective cells, is employed to determine the finite amplitude steady solutions. These solutions are represented in terms of convective modes whose amplitudes can be either small or of order unity. Stability of these solutions is investigated with respect to three dimensional disturbances. A variable gravity function introduces two non-dimensional parameters. For certain range of values of these two parameters, double or triple cellular structure in the vertical direction can be realized. Hexagonal patterns are preferred for sufficiently small amplitude of convection, while square patterns can become dominant for larger values of the convective amplitude. Variable gravity can also affect significantly the wavelength of the cellular pattern and the onset condition of the convective motion.
\end{abstract}

2000 Mathematics Subject Classification. Primary 76Exx, 76Rxx, 80Axx.

1. Introduction and formulation. This paper studies the problem of finite amplitude convection in a horizontal layer of infinite Prandtl number fluid of depth $d$ and bounded by two rigid plates subjected to a gravity function which varies quadratically with respect to the vertical variable $z$. Such a problem is of particular interest both in terms of fundamental knowledge and in terms of geological applications. Earth's mantle can be approximated as an infinite Prandtl number fluid because its viscosity is extremely large. In addition, the gravity field may vary as a function of radius only [4].

The present convection layer is assumed to be subjected to nearly insulating conditions at the top and bottom boundaries of the fluid layer. Such assumption is mainly for the mathematical convenience since, as was shown before [9], the governing system for such convection layer can be generalized and solved easily for cases where the coefficients of the terms in the governing equations vary with respect to the vertical variable $z$.

The present investigation is an extension of the small-amplitude theory to the finite-(but not necessarily small) amplitude regime, where the horizontal wave numbers $\alpha_{n}(n=1,2, \ldots)$ that contribute to the horizontal planform functions satisfy the relationship

$$
\alpha_{n}=\eta_{n} \gamma^{1 / 2}, \quad \gamma \equiv\left(\frac{\beta}{D}\right)^{2 / 3} \ll 1,
$$

where $d D$ is the dimensional thickness of either horizontal rigid plate boundary, $\beta$ is 
the ratio of thermal conductivity $\lambda^{e}$ of the boundary to thermal conductivity $\lambda$ of the fluid layer and the coefficients $\eta_{n}$ are assumed to be of the order unity and independent of $\gamma$. This finite amplitude theory was developed by Riahi [7] in the context of a Marangoni convection problem and the reader is referred to this reference for details of the theory.

We consider an infinite horizontal layer of fluid of depth $d$ bounded above and below by two infinite horizontal rigid plates of finite thickness $d D$ and thermal conductivity $\lambda^{e}$. In the steady static state, a constant heat flux transverses the system such that the temperatures $T_{0}$ and $T_{0}+\Delta T$ are attained at the upper and lower boundaries of the fluid. It is assumed that the gravity function $g G(z)$ varies quadratically with respect to the vertical variable $z$. Here the expression for $G(z)$ is normalized so that $\langle G(z)\rangle=1$, where the angular bracket indicates an average over the fluid layer. We shall define the Rayleigh number $R$ based on the constant $g$ which is the average of the gravity function.

It is convenient to use non-dimensional variables in which length, velocity, time, and temperature in the fluid flow are scaled respectively by $d, K / d, d^{2} / K$, and $q d / R$, where $q=\Delta T /[d(1+2 D / \beta)]$ is the negative temperature gradient in the fluid (in the absence of fluid motion) and $K$ is the thermal diffusivity of the fluid. Under the usual Boussinesq approximation, the non-dimensional forms of the equations for momentum, heat and conservation of mass can be simplified by using the representation

$$
\boldsymbol{u}=\boldsymbol{\delta} v=\nabla \times \nabla \times \hat{\boldsymbol{z}} v
$$

for the divergence-free velocity vector $\boldsymbol{u}$. Here $\hat{\boldsymbol{z}}$ represents the unit vector in the vertical direction and $v$ is the poloidal function for the velocity vector. The toroidal component $\nabla \times \hat{\boldsymbol{z}} \psi$ of $\boldsymbol{u}$ is not included in (1.2) since it can be shown that it is negligible in the limit of infinite Prandtl number $P_{r}$ for the present analysis. Using (1.2), the vertical component of the double curl of the momentum equation in the limit of $P_{r}=\infty$ and the heat equation yield the following equations

$$
\begin{gathered}
\Delta_{2}\left[\nabla^{4} v-G(z) \theta\right]=0, \\
\nabla^{2} \theta-R \Delta_{2} v=\frac{\partial \theta}{\partial t}+\boldsymbol{\delta} v \cdot \nabla \theta, \\
\frac{\partial \theta_{e}}{\partial t}=\mu \nabla^{2} \theta_{e}
\end{gathered}
$$

where $\theta$ and $\theta_{e}$ are the temperature fluctuations in the fluid layer and in the plates, $\mu=K_{e} / K$ is the ratio of the thermal diffusivity of the plates to that of the fluid, $R=a g q d^{4} / K v$ is the Rayleigh number, $a$ is the coefficient of thermal expansion, $v$ is the kinematic viscosity, $t$ is the time variable, and $\Delta_{2}$ is the horizontal Laplacian. The associated boundary conditions for $(1.3)[3,9]$ are

$$
v=\frac{\partial v}{\partial z}=0 \quad \text { at } z= \pm \frac{1}{2}
$$




$$
\begin{gathered}
\theta-\theta_{e}=\frac{\partial}{\partial z}\left(\theta-\beta \theta_{e}\right)=0 \quad \text { at } z= \pm \frac{1}{2}, \\
\theta_{e}=0 \quad \text { at } z= \pm\left(\frac{1}{2}+D\right) .
\end{gathered}
$$

Riahi [9] determined the boundary conditions for $\theta$ by solving (1.3c), (1.4b), and (1.4c), subjected to the restriction that the thickness $D$ of each plate is small in comparison to the horizontal dimension of the convection cells. Assuming the same restriction here, the boundary conditions for $\theta$ are [9]

$$
\frac{\partial \theta}{\partial z}=\mp \gamma^{2} \theta \text { at } z= \pm \frac{1}{2} .
$$

From previous studies [3, 8, 9] it is known that the predominant wave number in the horizontal direction vanishes when $\gamma$ tends to zero. For the investigation of this limit we use $\gamma$ as a perturbation parameter and anticipate that the relationship (1.1) holds in the limit of small $\gamma$.

In the next section, we present the steady convection based on the system (1.3a), (1.3b), (1.4a), and (1.5), where the gravity function is given by

$$
G(z)=\left(1-\frac{G_{2}}{12}\right)+G_{1} z+G_{2} z^{2}
$$

Here $G_{1}$ and $G_{2}$ are two constant parameters and $G(z)$ is normalized so that $\langle G(z)\rangle=1$. Figure 1.1 presents some graphs of $G(z)$ versus $z$ for cases where the extremum of $G(z)$ is a maximum, while Figure 1.2 presents some graphs of $G(z)$ versus $z$ for cases where the extremum of $G(z)$ is a minimum.

2. Steady convection. We start by introducing the horizontal planform function $w(x, y)$ that has the representation

$$
w(x, y)=\sum_{\substack{m=1, n=-N_{m}}}^{\substack{m=\infty, n=N m}} \varepsilon_{m} A_{n m} W_{n m}, \quad W_{n m}=\exp \left(i \boldsymbol{k}_{n m} \cdot \boldsymbol{r}\right),
$$

and the function $w_{m}(x, y)$ defined by

$$
w_{m}(x, y)=\sum_{n=-N_{m}}^{n=N_{m}} A_{n m} W_{n m}
$$

which satisfies the relation

$$
\Delta_{2} w_{m}+\alpha_{m}^{2} w_{m}=0, \quad\left\langle w_{m}^{2}\right\rangle=1
$$

Here $i$ is the imaginary number $\sqrt{-1}, \boldsymbol{r}$ is the position vector $(x, y), \varepsilon_{m}$ is the amplitude of the $m$ th mode and $\boldsymbol{k}_{n m}$ are the horizontal wave-number vectors for the $m$ th mode that satisfy the properties

$$
\boldsymbol{K}_{n m} \cdot \hat{\boldsymbol{z}}=0, \quad\left|\boldsymbol{K}_{n m}\right|=\alpha_{m}, \quad \boldsymbol{K}_{n m}=-\boldsymbol{K}_{-n m} .
$$




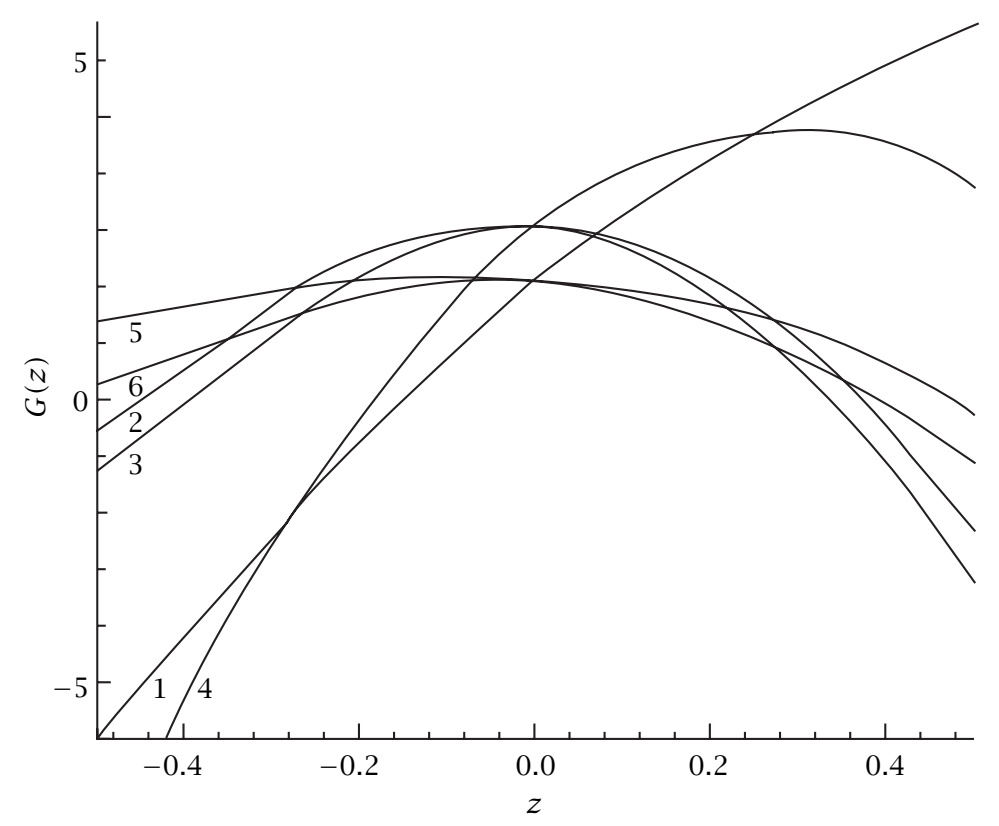

FIGURE 1.1. The gravity function $G(z)$ versus $z$ for $G_{2}<0$. Each curve is labeled by a number given just below the curve. The curves 2,3 , and 4 are for $G_{2}=-14.5$ and correspond, respectively, to $G_{1}=-5 / 3,0.0$, and 12.0. The curves 5,6 , and 1 are for $G_{2}=-3.0$ and correspond, respectively, to $G_{1}=-5 / 3,0.0$, and 12.0 .

The coefficients $A_{n m}$ satisfy the conditions

$$
\sum_{n=-N_{m}}^{n=N_{m}} A_{n m} A_{n m}^{*}=1, \quad A_{n m}^{*}=-A_{n m},
$$

where $N_{m}$ denotes the number of horizontal wave-number vectors $\boldsymbol{K}_{n m}$ participating in the $m$ th mode and the asterisk indicates the complex conjugate. The representation (2.1), (2.2), (2.3), and (2.4) given above are a generalization of representation in the small amplitude case [3] to those in the finite-(but not necessarily small) amplitude case.

The solutions of the steady state form of the governing system are obtained in terms of a series in powers of $\gamma$

$$
(v, \theta, R)=\sum_{n=0} \gamma^{n}\left(v_{n}, \theta_{n}, R_{n}\right)
$$

To $o(1)$, equations (1.3a) and (1.3b) and the boundary conditions (1.4a) and (1.5) yield solutions of the form

$$
\left(v_{0}, \theta_{0}\right)=\left[H_{0}(z), 1\right] w(x, y)
$$




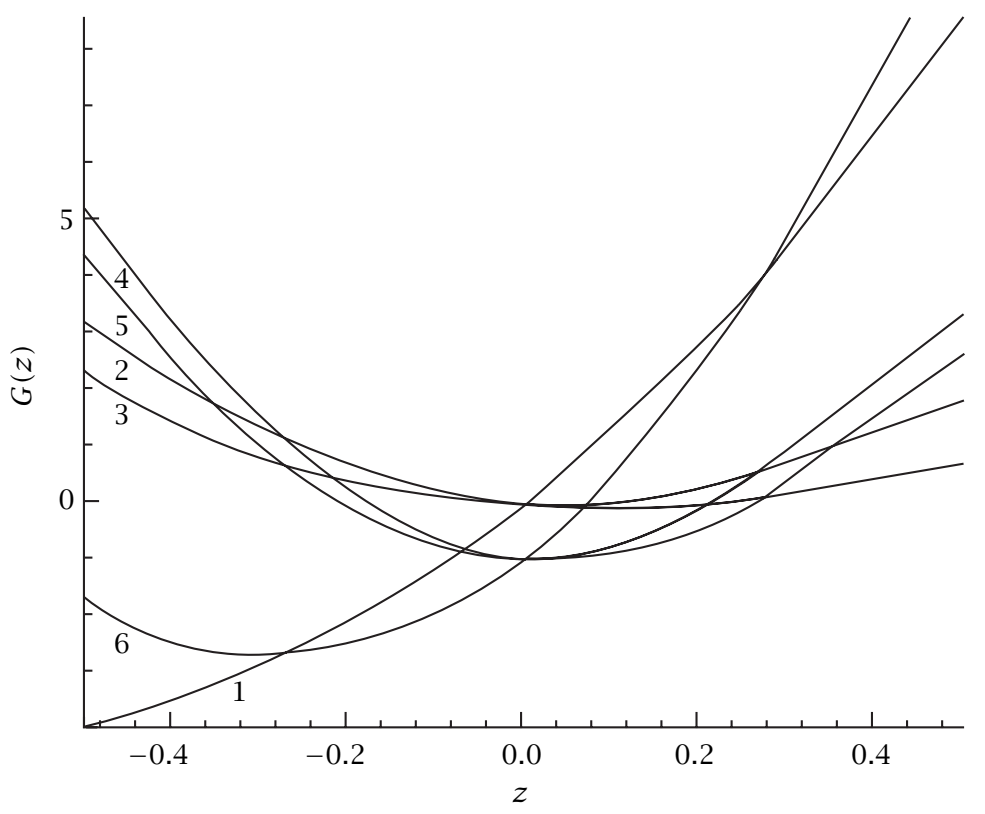

FIGURE 1.2. The gravity function $G(z)$ versus $z$ for $G_{2}>0$. Each curve is labeled by a number given just below the curve. The curves 2,3 , and 1 are for $G_{2}=8.5$ and correspond, respectively, to $G_{1}=-5 / 3,0.0$, and 12.0. The curves 4,5 , and 6 are for $G_{2}=20.0$ and correspond, respectively, to $G_{1}=$ $-5 / 3,0.0$, and 12.0 .

where

$$
H_{0}(z)=\frac{\left(z^{2}-(1 / 4)\right)^{2}\left[\left(G_{2} / 3\right) z^{2}+G_{1} z+\left(5-(1 / 4) G_{2}\right)\right]}{5 !} .
$$

In deriving the solutions a normalization condition of the form

$$
\left\langle\theta_{n} \theta_{0}\right\rangle=\delta_{n o} \sum_{m} \varepsilon_{m}^{2}
$$

has been assumed. This condition has been used to determine the solution $\theta_{n}$. Here $\delta_{n 0} \equiv 1$ for $n=0$ and zero otherwise.

The order $\gamma$ system for (1.3a), (1.3b), (1.4a), and (1.5) yield solutions $v_{1}$ and $\theta_{1}$. Averaging the equation for $\theta_{1}$ over the fluid layer, we find

$$
R_{0}=\frac{15120}{21-G_{2}}
$$

Here, $R_{0}$ has the value of 720 in the limit of $G_{2} \rightarrow 0$ in agreement with the constant gravity result [3]. Multiplying the order $\gamma^{2}$ equation for $\theta_{2}$ by $W_{n l}$, averaging over the 
fluid layer, and using order $\gamma^{2}$ boundary conditions, we find the following result:

$$
-2 \gamma \varepsilon_{l} A_{n l}^{*}+\left\langle W_{n l} \Delta_{2}\left[\theta_{1}-\left(R_{1} v_{0}+R_{0} v_{1}\right)\right]\right\rangle=\left\langle W_{n l}\left(\boldsymbol{\delta} v_{0} \cdot \nabla \theta_{1}+\boldsymbol{\delta} v_{1} \cdot \nabla \theta_{0}\right)\right\rangle \text {. }
$$

Using (2.6) and solutions $v_{1}$ and $\theta_{1}$ in (2.9), we find that (2.9) is a system of nonlinear algebraic equations for $R_{1}$ and the coefficients $A_{n l}\left(n=-N_{n}, \ldots,-1,1, \ldots, N_{n} ; l=\right.$ $1, \ldots, \infty)$ as functions of $N_{l}, \eta_{l}$, flow pattern, and amplitudes $\varepsilon_{l}$. This system generally admits many different irregular and regular solutions [7]. As in the case of small amplitude theory [3], we restrict our analysis to the regular cases where the flow pattern is in the form of either rolls $\left(N_{l}=1\right)$, squares $\left(N_{l}=2\right)$, or hexagons $\left(N_{l}=3\right)$. Hence we apply the usual algebra and procedure [9] for simplifying the system (2.4) and (2.9). For dominant mode of convection with wave number $\alpha_{l}$ [7], it leads to the following results:

$$
\begin{gathered}
\left|A_{n l}\right|^{2}=\frac{1}{\left(2 N_{l}\right)} \quad\left(l=1, \ldots ; n=1, \ldots, N_{l}\right), \\
R_{1}=R_{0}\left(2 \eta_{l}^{-2}+S_{l} \eta_{l}^{2}\right),
\end{gathered}
$$

where

$$
\begin{aligned}
S_{l}= & B_{0}+B_{1} \varepsilon_{l}\left(\delta_{3 N_{l}}\right)+B_{2} \varepsilon_{l}^{2} \\
B_{0}= & \frac{16.227-1.366 G_{2}-0.184 G_{1}^{2}+0.027 G_{2}^{2}}{\left(21-G_{2}\right)^{2}} \\
B_{1}= & -\frac{\sqrt{6} G_{1}\left(0.173+0.016 G_{2}\right)}{\left(21-G_{2}\right)}, \\
B_{2}= & \left\{\left(0.0334+0.0002 G_{1}^{2}+0.0002 G_{2}^{2}-0.0033 G_{2}\right)+\left(1-\delta_{1 N_{l}}\right)\right. \\
& \times \sum_{m=2}^{N_{l}}\left[\left(0.0125+0.0001 G_{1}^{2}+0.0001 G_{2}^{2}-0.0013 G_{2}\right)\right. \\
\left.\left.\quad+\left(0.0834+0.0002 G_{1}^{2}+0.0002 G_{2}^{2}-0.0080 G_{2}\right) \phi_{m 1}^{2}\right]\right\} /\left(15120 N_{l}\right) & \\
\phi_{m n}= & \frac{\left(\boldsymbol{K}_{m l} \cdot \boldsymbol{K}_{n l}\right)}{\alpha_{l}^{2}}
\end{aligned}
$$

and $\delta_{3 N_{l}}$ is a Kronecker delta so that it equals one for $N_{l}=3$ and zero otherwise. Minimizing the expression (2.10b) for $R_{1}$ with respect to $\eta_{l}$ yields

$$
R_{1 p}=2 R_{0}\left(2 S_{l}\right)^{1 / 2}, \quad \eta_{p}=\left(\frac{2}{S_{l}}\right)^{1 / 4},
$$

where $\eta_{p}$ designates the preferred $\eta_{l}$ at which $R_{1}$ is minimized to $R_{1 p}$ with respect to the scaled wave number $\eta_{l}$. Using the approximate expression

$$
R_{1}=\frac{R-R_{0}}{\gamma}
$$


for $R_{1}$ in (2.10b), we obtain the functional relationship between amplitude and wave number for the dominant mode and for given $R, G_{1}$ and $G_{2}$. Using (2.12) in the expression for $R_{1 p}$ given in (2.11), we obtain the expression for the preferred amplitude $\varepsilon$ as a function of $R, G_{1}$, and $G_{2}$.

It should be noted from (2.10c) that depending on the sign of $\varepsilon_{l}$, the expression for $S_{l}$ can either be given by the so-called subcritical form [7]

$$
S_{l}^{-}=B_{0}-\left|B_{1}\right|\left|\varepsilon_{l}\right| \delta_{3 N_{l}}+B_{2} \varepsilon_{l}^{2}
$$

or by the so-called supercritical form [7]

$$
S_{l}^{+}=B_{0}+\left|B_{1}\right|\left|\varepsilon_{l}\right| \delta_{3 N_{l}}+B_{2} \varepsilon_{l}^{2}
$$

Here $S_{l}^{-}<S_{l}^{+}$unless $N_{l} \neq 3$ or $\left|B_{1}\right|=0$. Hence $R_{1 p}$ and $\eta_{p}$ are given in terms of $S_{l}^{-}$. The expressions for $S_{l}^{-}$and $S_{l}^{+}$are called subcritical and supercritical, respectively, with reference to the expression $B_{0}+B_{2} \varepsilon_{l}^{2}$. Also, the flow due to the dominant mode is called subcritical flow if $S_{l}=S_{l}^{-}$and supercritical flow if $S_{l}=S_{l}^{+}$[7].

For either subcritical or supercritical flow case, the corresponding flow pattern is that due to hexagonal cells, and we can find the sign of the vertical motion, at any plane $z=z_{0},\left|z_{0}\right|<1 / 2$, at the cells' center $\boldsymbol{r}=0$ by the following procedure. We have

$$
u_{3}=\boldsymbol{u} \cdot \hat{z} \simeq \frac{\gamma}{\sqrt{6}} H_{0}\left(z_{0}\right) \varepsilon_{l} \eta_{l}^{2} \quad \text { at } \boldsymbol{r}=0, z=z_{0} .
$$

Hence, $u_{3}$ at $z=z_{0}$ and $\boldsymbol{r}=0$ has the same sign as $H_{0} \varepsilon_{l}$. Since the minimum state (2.11) is due to the subcritical flow for $N_{l}=3$, the subcritical hexagons are preferred over the supercritical hexagons. If $u_{3}$ given by (2.14) is negative, then the subcritical hexagons are called down-hexagons, while for $u_{3}>0$ such hexagons are called uphexagons.

Using the approximate expression

$$
H_{c}=\left\langle\theta u_{3}\right\rangle \simeq-\left\langle\theta_{0} \Delta_{2} v_{0}\right\rangle=\gamma \varepsilon_{l}^{2} \eta_{l}^{2}
$$

for the heat transported by convection, the results (2.11) and (2.12) can be used to determine the expression for $H_{c}$ as function of $R, \gamma, G_{1}$ and $G_{2}$. For the case of convection in the form of two-dimensional rolls, $N_{l}=1$, we find

$$
H_{c}=\frac{4\left|R_{0}\right| \gamma^{2}}{\left|\left(R-R_{0}\right)\right|}\left[\frac{\left(R-R_{0}\right)^{2}}{8 R_{0}^{2} \gamma^{2}}-B_{0}\right]\left(\frac{1}{B_{3} / 2+B_{4}}\right),
$$

where

$$
\begin{aligned}
& B_{3}=\frac{0.0417+0.0001 G_{1}^{2}+0.0001 G_{2}^{2}-0.0040 G_{2}}{15120}, \\
& B_{4}=\frac{0.0125+0.0001 G_{1}^{2}+0.0001 G_{2}^{2}-0.0013 G_{2}}{15120} .
\end{aligned}
$$


For the case of square pattern convection, $N_{l}=2$, we find

$$
H_{c}=\frac{4\left|R_{0}\right| \gamma^{2}}{\left|\left(R-R_{0}\right)\right|}\left[\frac{\left(R-R_{0}\right)^{2}}{8 R_{0}^{2} \gamma^{2}}-B_{0}\right]\left(\frac{1}{B_{3} / 4+B_{4}}\right)
$$

Detailed computations indicate that $B_{3}=\left\langle H_{0}^{2}\right\rangle$, so that $B_{3}>0$ as (2.16b) also indicates. Hence, comparing (2.16a) and (2.17), we find that square cells transport more heat than rolls for all possible values of $G_{1}$ and $G_{2}$. For the case of hexagon pattern convection, $N_{l}=3$, we find the following result for the preferred subcritical hexagons:

$$
H_{c}=\frac{\left|R_{0}\right| \gamma^{2}}{B_{5}^{2}\left|\left(R-R_{0}\right)\right|}\left\{\left|B_{1}\right|+\frac{B_{5}}{\left|B_{5}\right|} \sqrt{B_{1}^{2}+4 B_{5}\left[\frac{\left(R-R_{0}\right)^{2}}{8 R_{0}^{2} \gamma^{2}}-B_{0}\right]}\right\}^{2}, \quad B_{5} \equiv \frac{B_{3}}{2}+B_{4}
$$

Using (2.11) and (2.12), we obtain the following expression for the preferred wavelength $L_{p}=2 \pi /\left(\eta_{p} \sqrt{\gamma}\right)$ :

$$
L_{p}=\frac{\pi \sqrt{\left|R-R_{0}\right|}}{\gamma \sqrt{\left|R_{0}\right|}}
$$

3. Stability analysis. We now investigate the stability of the steady solutions that were determined in the previous section. The equations for the time dependent disturbances $\tilde{v}$ and $\tilde{\theta}$ are given by

$$
\begin{gathered}
\Delta_{2}\left(\nabla^{4} \tilde{v}-G \tilde{\theta}\right)=0 \\
\left(\nabla^{2}-\sigma\right) \tilde{\theta}-R \Delta_{2} \tilde{\theta}=\boldsymbol{\delta} \tilde{v} \cdot \nabla \theta+\boldsymbol{\delta} v \cdot \nabla \tilde{\theta}
\end{gathered}
$$

where the growth rate $\sigma$ is defined by $\partial / \partial t=\sigma$. The boundary conditions for $\tilde{v}$ and $\tilde{\theta}$ are the same as those for $v$ and $\theta$, respectively, so that

$$
\boldsymbol{v}=\frac{\partial \boldsymbol{v}}{\partial z}=\left(\frac{\partial}{\partial z} \pm \gamma^{2}\right) \tilde{\theta}=0 \quad \text { at } z= \pm \frac{1}{2}
$$

The system (3.1) is solved by using the following perturbation expansion:

$$
(\tilde{v}, \tilde{\theta}, \sigma)=\sum_{n=0} \gamma^{n}\left(\tilde{v}_{n}, \tilde{\theta}_{n}, \sigma_{n}\right)
$$

To $o$ (1) equations (3.1a), (3.1b) and the boundary conditions (3.1c) are of the same form as the corresponding ones for the steady finite amplitude case. The solutions are

$$
\tilde{v}_{0}=H_{0}(z) \tilde{w}(x, y), \quad \tilde{\theta}_{0}=\tilde{w}(x, y), \quad \sigma_{0}=0,
$$


where

$$
\tilde{w}(x, y)=\sum_{n=-\infty}^{n=\infty} \tilde{A}_{n} \tilde{w}_{n}, \quad \tilde{w}_{n}=\exp \left(i \tilde{\boldsymbol{k}}_{n} \cdot \boldsymbol{r}\right) .
$$

Here, $\tilde{w}(x, y)$ is the horizontal planform function of the general three-dimensional disturbances, $\tilde{A}_{n}$ are constant coefficients, $\tilde{\boldsymbol{k}}_{n}$ are the horizontal wave number vectors of disturbances which satisfy the properties

$$
\tilde{\boldsymbol{k}}_{n} \cdot \hat{z}=0, \quad\left|\tilde{\boldsymbol{k}}_{m}\right|=\tilde{\alpha}_{n}-\tilde{\eta}_{n} \gamma^{1 / 2}, \quad \tilde{\boldsymbol{k}}_{n}=-\tilde{\boldsymbol{k}}_{-n},
$$

and the parameters $\tilde{\eta}_{n}$ are assumed to be at most of the order unity and independent of $\gamma$.

The order $\gamma$ system for the governing equations (3.1a), (3.1b) and boundary conditions (3.1c) yields solutions $\tilde{v}_{1}$ and $\tilde{\theta}_{1}$. The solvability condition for the order $\gamma$ system then yields

$$
\sigma_{1}=0
$$

Multiplying the order $\gamma^{2}$ equation for $\tilde{\theta}_{2}$ by $\tilde{w}_{n}$, averaging over the fluid layer, and using order $\gamma^{2}$ boundary conditions, we find the following result:

$$
\begin{aligned}
-\gamma A_{n}^{*}\left(2+\sigma_{2}\right)+\left\langle\tilde{w}_{n} \Delta_{2}\left[\tilde{\theta}_{1}-\left(R_{1} \tilde{v}_{0}+R_{0} \tilde{v}_{1}\right)\right]\right\rangle \\
=\left\langle\tilde{w}_{n}\left[\boldsymbol{\delta} \tilde{v}_{0} \cdot \nabla \theta_{1}+\boldsymbol{\delta} v_{0} \cdot \nabla \tilde{\theta}_{1}+\boldsymbol{\delta} \tilde{v}_{1} \cdot \nabla \theta_{0}+\boldsymbol{\delta} v_{1} \cdot \nabla \tilde{\theta}_{0}\right]\right\rangle .
\end{aligned}
$$

Using (2.6), (3.3), and the solution $v_{1}, \theta_{1}, \tilde{v}_{1}$, and $\tilde{\theta}_{1}$ in (3.5), we find that (3.5) is a system of algebraic equations for $\sigma_{2}$ and the coefficients $\tilde{A}_{n}$. Here, the procedure to determine the growth rates $\sigma_{2}$ is similar to those used in [1] and [9]. Rather than repeating that procedure for deriving the eigenvalues $\sigma_{2}$ of (3.5), we refer the reader to these references for further details. Following Riahi [9], we find that the only possible stable solutions are those of subcritical hexagons and squares. Square pattern convection is found to be stable for

$$
\left|\varepsilon_{l}\right| \geq \frac{12\left|B_{1}\right|}{\sqrt{6} B_{3}}
$$

while subcritical hexagon pattern convection is found to be stable for

$$
\left|\varepsilon_{l}\right| \geq \frac{2\left|B_{1}\right|}{\left|\left(B_{3}+2 B_{4}\right)\right|} .
$$

In addition, present analysis is valid, provided

$$
\left|\varepsilon_{l}\right| \ll \gamma^{-1}
$$

(see [3]).

4. Discussion of the results. Before discussing the results obtained in the last two sections, it is of interest to discuss the structure of the quadratic form of the gravity function $G(z)$ given by (1.6). Due to such quadratic form of $G(z)$, we assume that

$$
G_{2} \neq 0 \text {. }
$$


The function $G(z)$ has an extremum at

$$
z=-\frac{G_{1}}{2 G_{2}} .
$$

This extremum is a minimum for

$$
G_{2}>0
$$

and is a maximum for

$$
G_{2}<0 \text {. }
$$

The extremum of $G(z)$ lies in the layer interval $|z|<1 / 2$ for

$$
-1<-\frac{G_{1}}{G_{2}}<1
$$

The function $G(z)$ is symmetric with respect to its extremum value if

$$
G_{1}=0
$$

and asymmetric otherwise. See Figures 1.1 and 1.2 which agree with the above analytical features.

The $z$-dependence $H_{0}(z)$ of the leading order term for the vertical component $u_{3}=$ $-\Delta_{2} v_{0}$ of the velocity vector, given by (2.6b) indicate that it can vanish once or twice within the layer interval $|z|<1 / 2$, provided

$$
\left|-\left(\frac{3 G_{1}}{G_{2}}\right)+\left[9\left(\frac{G_{1}}{G_{2}}\right)^{2}+\left(3-\frac{60}{G_{2}}\right)\right]^{1 / 2}\right|<1
$$

and/or

$$
\left|-\left(\frac{3 G_{1}}{G_{2}}\right)-\left[9\left(\frac{G_{1}}{G_{2}}\right)^{2}+\left(3-\frac{60}{G_{2}}\right)\right]^{1 / 2}\right|<1 .
$$

The fluid layer can then be composed of double or triple cell structures in the vertical direction with different flow direction in each set of cells at given $\boldsymbol{r}$ values. For example, double-layer structure can exist for $G_{1}=12$ and $G_{2}=-3$. (Figure 1.1), while triple-layer structure can exist for $G_{1}=-5 / 3$ and $G_{2}=20$ (Figure 1.2).

The expression (2.8) for $R_{0}$ indicates that the effect of $G_{2}$ is destabilizing for $G_{2}<0$ and stabilizing for $G_{2}>0$, and $R_{0}$ is independent of $G_{1}$. The expressions (2.10b), (2.10c), and (2.12) indicate the functional relationship between $\varepsilon_{l}$ and $\eta_{l}$ for given $R, \gamma, G_{1}$ and $G_{2}$. Thus the dominant mode with certain amplitude allows particular wavelength. Of particular interest is the preferred mode represented by (2.11) where the expression for $R_{1}$ is minimized with respect to the wavelength. Using (2.10c), (2.11), and (2.12), we find that the amplitude $\left|\varepsilon_{p}\right|$ for the preferred mode can increase with $R$, for given $\gamma, G_{1}$ and $G_{2}$, provided

$$
\frac{\left[\left|B_{1}\right| \delta_{3 N_{l}}+\left(R-R_{0}\right) /\left(2 \gamma R_{0}\right)^{2}\right]}{B_{2}}>0 .
$$

Furthermore, (2.10c) and (2.11) indicate that the preferred wavelength of the convection cells depend strongly on the gravity parameters $G_{1}$ and $G_{2}$. For example, for small 
$\left|\varepsilon_{l}\right|$ case, $\eta_{p}$ is smaller than the corresponding value 3.006, which is realized in the absence of variable $G\left(G_{1}=G_{2}=0\right)$, provided $\left|G_{1}\right|$ is sufficiently small and $G_{2}$ lies in the range

$$
0<G_{2}<18.312 .
$$

However, if $\left|G_{2}\right|$ is sufficiently small and $\left|G_{1}\right|$ is sufficiently large, then $\eta_{p}>3.006$. It should be noted from (2.10c) and (2.11) that the present theory breaks down if $S_{l}$ becomes negative. Hence, the condition for the validity of the present results is that

$$
B_{2}\left|\varepsilon_{l}\right|^{2}-\left|B_{1}\right|\left|\varepsilon_{l}\right| \delta_{3 N_{l}}+B_{0}>0 .
$$

The expression for $R_{1 p}$ in (2.11) indicates that there is no finite amplitude instability for $R_{0}>0$, but there is such instability for $R_{0}<0$. However, for the small amplitude case, stable flow can be shown to be subcritically operative. Such subcritical behavior is found to exist for hexagons if either

$$
G_{1}\left(2.076+0.192 G_{2}\right)<0 \text { for } \varepsilon_{l}<0
$$

or

$$
G_{1}\left(2.076+0.192 G_{2}\right)>0 \text { for } \varepsilon_{l}>0 .
$$

Similar subcritical behavior is exhibited by squares if

$$
G_{2}>25.3+6.24\left(1+0.18 G_{1}^{2}\right)^{1 / 2} .
$$

Hence subcritical instability can exist only in a small range of $R$ where amplitude of motion is sufficiently small. For large amplitudes second order terms in $\varepsilon_{l}$ in (2.10c) dominates over lower order terms in $\varepsilon_{l}$ resulting in a positive $R_{1}$ given by (2.10b).

To discuss the direction of motion at the cells' center in a sub-layer where $H_{0}(z)$ has only one sign, we restrict the discussion for the hexagonal cells only since it is known [2] that a change of sign of motion at the cells' center can lead to qualitatively different cellular structures only in the case of hexagons. Thus we consider the expression (2.14) for $u_{3}$ at $z=z_{0}\left(\left|z_{0}\right|<(1 / 2)\right)$ and note that it satisfies the condition

$$
u_{3} H_{0}(z) \varepsilon_{l}>0
$$

We already found that stable subcritical hexagons are those for which the condition

$$
B_{1} \varepsilon_{l}<0 .
$$

Given $G_{1}$ and $G_{2}, B_{1}$ has a definite sign implying that $\varepsilon_{l}$ has one sign opposite to that of $B_{1}$. Thus, it follows that $H_{0}(z)$ has a definite sign. Consequently, the sign of $u_{3}$ can be found easily. For example, if $z_{0}=0,0<G_{2}<20$ and $G_{1}>0$, then $H_{0}\left(z_{0}\right)>0$, $B_{1}<0$ and $\varepsilon_{l}>0$. Hence $u_{3}>0$ and motion is upward at the hexagons' center and at the mid-plane $z=0$. For $z_{0}=0$ and $0<G_{2}<20$ and $G_{1}<0$, then $H_{0}\left(z_{0}\right)>0, B_{1}>0$ and $\varepsilon_{l}<0$. Hence $u_{3}<0$ and motion is downward at the hexagons' center and at the mid-plane $z=0$. The results for the sign of motion appears to be independent of the magnitudes of the amplitudes. For the case $B_{1}=0$, the flow direction can be discussed if $S_{l}$ is modified by inclusion of the results to the order $\gamma^{3}$ in the governing equations. 
The expressions (2.16), (2.17), and (2.18) for heat transported by convection, due to rolls, squares and hexagons, provide dependence of the heat flux on $R, \gamma, G_{1}$ and $G_{2}$. Since $\eta_{l}$ and $R_{1}$ change abruptly if the flow pattern changes, we expect that the heat flux changes abruptly if the convection pattern changes due to a bifurcation. Also a non-monotonicity of the heat flux with respect to $G_{1}$ and $G_{2}$ for a given $R$ cannot be ruled out.

The preferred wavelength $L_{p}$ of the stable convection cells given by (2.19) indicate that $L_{p}$ increases with $R$ for given $G_{2}$. It is independent of $G_{1}$ since $R_{0}$ does not depend on $G_{1}$.

The stability conditions (3.6a), (3.6b) indicate that although small amplitude theory may be adequate for small $\left|G_{1}\right|$ where the right-hand sides of (3.6a) and (3.6b) can become small compared to unity, it certainly is not adequate for large $\left|G_{1}\right|$ which require at least order one values for $\left|\varepsilon_{l}\right|$.

It can be deduced from the expression (2.10f) for $B_{2}$ that $B_{2}>0$ for both stable squares and subcritical hexagons. Using this result and the stability conditions (3.6a), (3.6b) in the expression (2.10c) for $S_{l}$, we can compare $\eta_{p}$, given in (2.11), to the corresponding $\eta_{c}$, defined by

$$
\eta_{c}=\left(\frac{2}{B_{0}}\right)^{1 / 4}
$$

which is the critical $\eta$ at which convection first occurs as $R$ is increased. We find

$$
\eta_{p}<\eta_{c}
$$

and note that $\left|\eta_{p}-\eta_{c}\right|$ increases with $\varepsilon_{l}$, and $\eta_{p}$ is very close to $\eta_{c}$ for small amplitude case. These results are in agreement with the corresponding ones obtained by Proctor [5] and Riahi [7] in the case of uniform gravity $(G \equiv 1)$.

Small amplitude convection with variable properties and internal heating was investigated by Riahi [6] using small amplitude theory of Busse and Riahi [3]. Riahi finds, in particular, that his general qualitative results depend on the symmetries and antisymmetries of the variable properties and internal heating functions with respect to the mid-plane of the fluid layer. Results of the present investigation are in general agreement with his results regarding unstable rolls and possible stable squares or hexagons.

\section{REFERENCES}

[1] F. H. Busse, The stability of finite amplitude cellular convection and its relation to an extremum principle, J. Fluid Mech. 30 (1967), 625-649. Zbl 159.28202.

[2] _ Nonlinear properties of thermal convection, Rep. Prog. Phys. 41 (1978), 1929-1967.

[3] F. H. Busse and N. Riahi, Nonlinear convection in a layer with nearly insulating boundaries, J. Fluid Mech. 96 (1980), 243-256. Zbl 429.76055.

[4] S. Chandrasekhar, Hydrodynamic and Hydromagnetic Stability, The International Series of Monographs on Physics, Clarendon Press, Oxford, 1961. MR 23\#B1270. Zbl 142.44103.

[5] M. R. E. Proctor, Planform selection by finite-amplitude thermal convection between poorly conducting slabs, J. Fluid Mech. 113 (1981), 469-485. MR 83b:76039. Zbl 484.76088.

[6] D. N. Riahi, Nonlinear convection with variable properties and internal heating, J. Math. Phys. Sci. 22 (1988), no. 2, 161-180. Zbl 642.76101. 
[7] _ Hexagon pattern convection for Benard-Marangoni problem, Int. J. Eng. Sci. 27 (1989), no. 6, 689-700. Zbl 685.76036.

[8] N. Riahi, Nonlinear convection in a porous layer with finite conducting boundaries, J. Fluid Mech. 129 (1983), 153-171. Zbl 559.76090.

[9] _ Nonlinear convection in a horizontal layer with an internal heat source, J. Phys. Soc. Japan 53 (1984), no. 12, 4169-4178. MR 86c:76029.

D. N. Riahi: Department of Theoretical and ApPlied Mechanics, 216 Talbot LaboRATORY, 104 S. WRIGHT STREET, UNIVERSITY OF ILLINOIS AT URBANA-CHAMPAIGN, URbANA, IL 61801, USA

Albert T. HSui: Department of GeOlogy, 245 Natural History Building, 1301 W. GREEN ST., UNIVERSITY OF ILLINOIS AT URBANA-ChAMPAIGN, URBANA, IL 61801, USA 


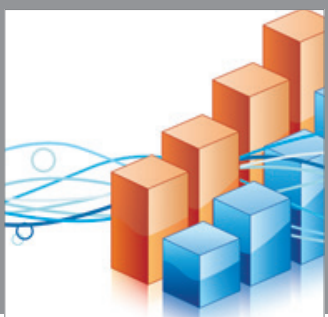

Advances in

Operations Research

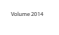

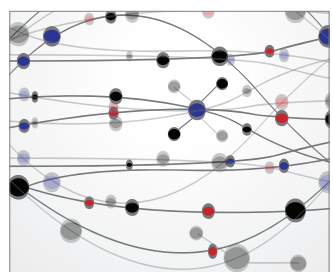

\section{The Scientific} World Journal
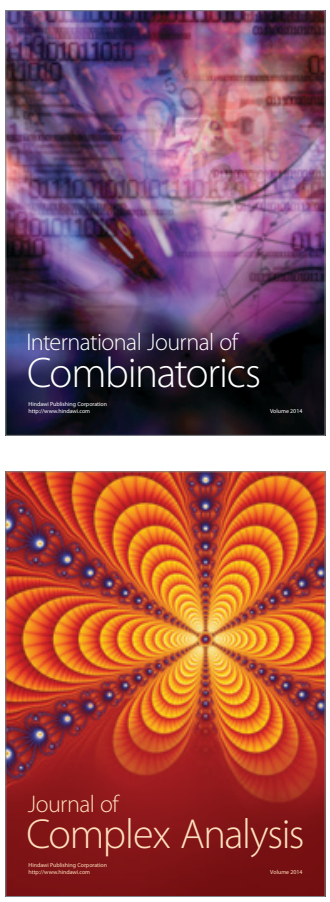

International Journal of

Mathematics and

Mathematical

Sciences
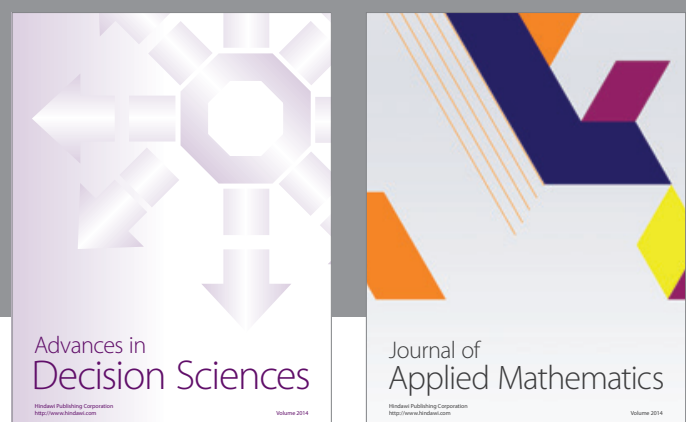

Journal of

Applied Mathematics
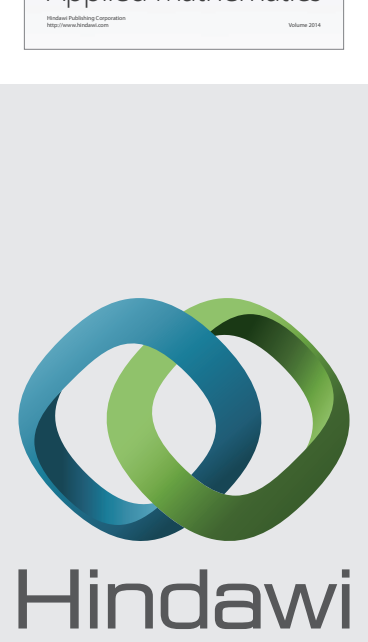

Submit your manuscripts at http://www.hindawi.com
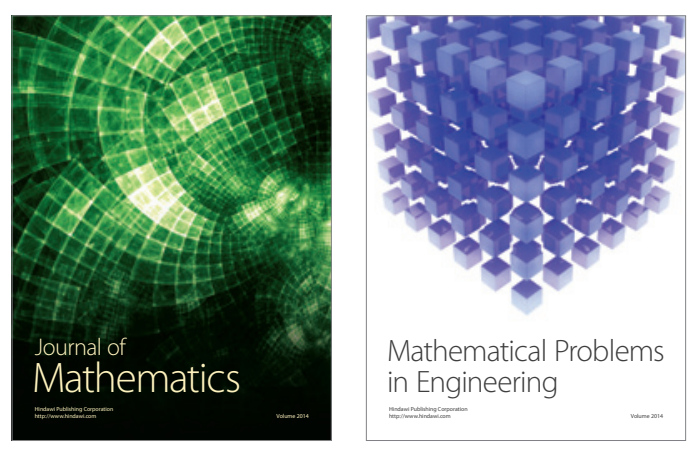

Mathematical Problems in Engineering
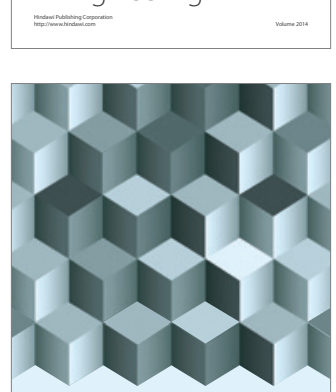

Journal of

Function Spaces
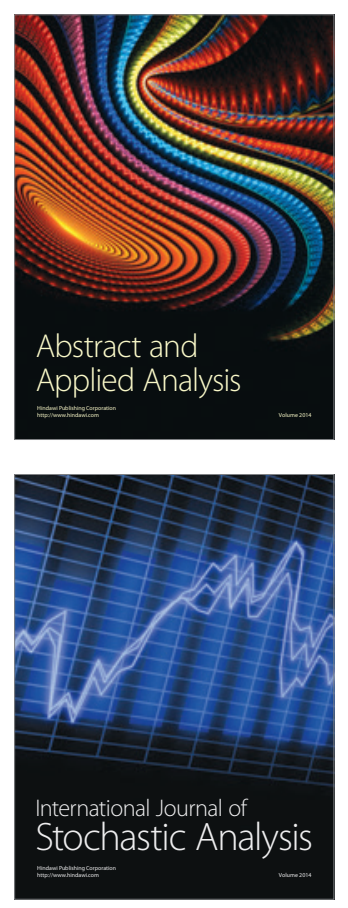

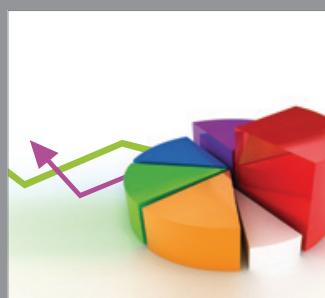

ournal of

Probability and Statistics

Promensencen
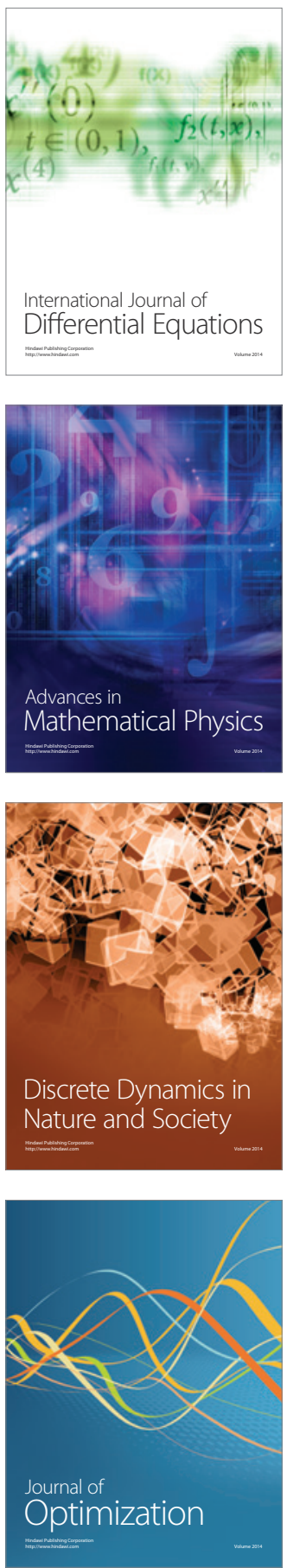